\title{
Author's Reply
}

\section{To the Letter to the Editor by Sorscher}

The comments of Dr. Sorscher underscore the challenges of working with limited data and rare scenarios in HER2 testing.

A few points deserve additional clarification. HER2 in situ hybridization (ISH) results that fall in the Group 2 category (those with HER2/CEP17 ratio $\geq 2.0$ and $<4$ mean HER2 signals/cell) are not considered negative without further consideration. Rather, the 2018 ASCO/CAP HER2 Testing in Breast Cancer guidelines update recommends that a concurrent HER2 immunohistochemistry $(\mathrm{IHC})$ result be used to help determine the overall HER2 status. ${ }^{1,2}$ If the $\mathrm{IHC}$ result is $3+$, it would be considered a positive result (based on concurrent IHC and $\mathrm{ISH}$ results), whereas if the $\mathrm{IHC}$ is 0 to $2+$ it would be considered HER2-negative overall. A standardized report comment describing the limitations of the data is also recommended to be included in these HER2 reports to recognize that this is a rare scenario with some uncertainties. This group was reexamined by the guidelines committee because of concern that using the HER2/CEP17 ratio alone in cases with low mean HER2 counts was resulting in a false-positive ISH HER2 status that was frequently discordant with concurrent IHCnegative results. While recognizing that ratio $\geq 2.0$-regardless of average HER2 copy number-was included in the original eligibility for the first adjuvant trials of trastuzumab, more recent data on Group 2 cases show that many of these cases are in fact HER2 IHC-negative, estrogen receptor-positive and can be low grade. ${ }^{3-5}$ In fact, $91 \%$ of Group 2 cases in the BCIRG005 and -006 trials tested for HER2 IHC were $\mathrm{IHC}$-negative $(0-1+)^{3,4}$ Published data from laboratories that dual-test routinely by IHC and fluorescence ISH (rather than only reflex ISH testing after a $2+\mathrm{IHC}$ result) also support that it is very uncommon for Group 2 ISH cases to have HER2 protein overexpression, but since it can occur, the IHC result can be used to help resolve the overall HER2 status of these cases. $^{3-5}$ Unlike the more common Group 1 ISH cases (ratio $>2.0$ and mean HER2 signals/cell $\geq 4$ ), Group 2 cases are a much less uniform group and can have a spec- trum of grades, hormone receptor status, and HER2 IHC results. Some cases are complex, and therefore repeat testing or expert consultation may be appropriate in some settings to provide additional information of the HER2 status when in question. NCCN recognizes these same HER2 testing principles for HER2 Group 2 ISH cases in the "Principles of Biomarker Testing: HER2 Testing" section of the NCCN Clinical Practice Guidelines in Oncology for Breast Cancer (page BINV-A; available at NCCN.org). We agree that the discussion section of these guidelines (page MS-4) needs to be updated to match these other updates to the guidelines.

We also agree that robust clinical data are frequently limited in rare scenarios. Some of the limited but more granular clinical trial data we do have about Group 2 ISH cancers are from those that accrued to the BCIRG-006 trial using adjuvant trastuzumab. A retrospective assessment of potential benefit from trastuzumab in this group failed to reach statistical significance to completely rule out a benefit, but the observed hazard ratio estimate was slightly greater that 1.0, favoring no trastuzumab benefit. ${ }^{3,4}$ Further confirmation of a lack of benefit with additional clinical trial data would of course be ideal, but given the rarity of these cases (reported in $0.4 \%-3.7 \%$ of cases) and the variability of trial entry criteria and specific HER2 data collected, it will be a challenge to achieve more significant results with a meta-analysis. ${ }^{2}$ As was recently pointed out in Johnston et al's reply ${ }^{6}$ to Dr. Sorscher's letter ${ }^{7}$ referencing their Journal of Clinical Oncology publication on the ALTERNATIVE trial (examining dual HER2 blockade with lapatinib and trastuzumab + aromatase inhibitor in HER2positive/hormone receptor-positive metastatic breast cancer), even some of the most recent clinical trial data frequently lack the specific HER2 testing data to accurately define a Group 2, HER2-negative population to determine efficacy in this subgroup. ${ }^{8}$ We support the interest in clinical trials collecting these data in a way that can be used to further study this and other uncommon HER2 ISH groups so that ideal treatment options can be better defined in these scenarios. Excluding these uncommon groups from trials also contributes to these clinical data gaps.
We have come a long way, in that now we are able to recognize and standardize how these uncommon HER2 result groups are defined, confirmed, and managed. However, the gray/data-limited zones do continue to exist and present challenges to practice.

Kimberly H. Allison, $M D^{a}$

\section{${ }^{a}$ Stanford Comprehensive Cancer Center, Stanford, California Email: allisonk@stanford.edu}

doi: 10.6004/jnccn.2021.7060

\section{References}

1. Wolff AC, Hammond MEH, Allison $\mathrm{KH}$, et al. Human epidermal growth factor receptor 2 testing in breast cancer: American Society of Clinical Oncology/College of American Pathologists clinical practice guideline focused update. J Clin Oncol 2018;36:2105-2122.

2. Gradishar WJ, Anderson BO, Abraham J, et al. NCCN Clinical Practice Guidelines in Oncology: Breast Cancer. Version 1.2021. Accessed April 23, 2021. To view the most recent version, visit NCCN.org

3. Press MF, Sauter G, Buyse M, et al. HER2 gene amplification testing by fluorescent in situ hybridization (FISH): comparison of the ASCOCollege of American Pathologists Guidelines with FISH scores used for enrollment in breast cancer international research group clinical trials. J Clin Oncol 2016;34:3518-3528.

4. Press MF, Villalobos I, Santiago A, et al Assessing the new American Society of Clinical Oncology/College of American Pathologists guidelines for HER2 testing by fluorescence in situ hybridization: experience of an academic consultation practice. Arch Pathol Lab Med 2016;140:1250-1258.

5. Ballard M, Jalikis F, Krings G, et al. 'Nonclassical' HER2 FISH results in breast cancer: a multi-institutional study. Mod Pathol 2017;30:227-235.

6. Sorscher S. Patients with group2, HER2-negative tumors on ALTERNATIVE. J Clin Oncol 2021;39:1188.

7. Johnston SRD, Gradishar WJ. Reply to S. Sorscher. J Clin Oncol 2021;39:1188-1189.

8. Johnston SRD, Hegg R, Im SA, et al. Phase III, randomized study of dual human epidermal growth factor receptor 2 (HER2) blockade with lapatinib plus trastuzumab in combination with an aromatase inhibitor in postmenopausal woman with HER2-positive, hormone receptor-positive metastatic breast cancer: updated results of ALTERNATIVE. J Clin Oncol 2021;39:79-89.

\section{CALL FOR CORRESPONDENCE}

JNCCN is committed to providing a forum to enhance collaboration between academic medicine and the community physician. We welcome comments about the NCCN Guidelines, articles published in the journal, or any other topic relating to cancer prevention, detection, treatment, supportive care, or survivorship. Please submit correspondence to www.editorialmanager.com/JNCCN.

Letters should be no more than 400 words, with no more than 5 references if included. NCCN reserves the right not to publish correspondence for any reason it deems appropriate. All letters are subject to editing and/or abridgment. 